Published in final edited form as:

Cancer. 2010 April 15; 116(8): 2001-2010. doi:10.1002/cncr.24939.

\title{
Occupational Sunlight Exposure and Risk of Renal Cell Carcinoma
}

\author{
Sara Karami, MPH, \\ Division of Cancer Epidemiology and Genetics, National Cancer Institute, NIH, DHHS, Bethesda, \\ MD, USA \\ Paolo Boffetta, Ph.D., \\ International Agency for Research on Cancer, Lyon, France \\ Patricia Stewart, Ph.D., \\ Stewart Exposure Assessments, LLC, Arlington, VA, USA, Formerly of the Division of Cancer \\ Epidemiology and Genetics, National Cancer Institute, Bethesda, MD, USA \\ Nathaniel Rothman, MD, \\ Division of Cancer Epidemiology and Genetics, National Cancer Institute, NIH, DHHS, Bethesda, \\ MD, USA
}

Katherine L. Hunting, Ph.D.,

Department of Epidemiology and Biostatistics, George Washington University, Washington, DC, USA

Mustafa Dosemeci, Ph.D.,

Division of Cancer Epidemiology and Genetics, National Cancer Institute, NIH, DHHS, Bethesda, MD, USA

\section{Sonja I. Berndt, Ph.D.,}

Division of Cancer Epidemiology and Genetics, National Cancer Institute, NIH, DHHS, Bethesda, MD, USA

\section{Paul Brennan, Ph.D.,}

International Agency for Research on Cancer, Lyon, France

\section{Wong-Ho Chow, Ph.D.,}

Division of Cancer Epidemiology and Genetics, National Cancer Institute, NIH, DHHS, Bethesda, MD, USA

\section{Lee E. Moore, Ph.D.,}

Division of Cancer Epidemiology and Genetics, National Cancer Institute, NIH, DHHS, Bethesda, MD, USA

\section{David Zaridze,}

Mukeria for the Institute of Carcinogenesis for Cancer Research Centre in Moscow, Russia

\section{Anush Mukeria,}

Mukeria for the Institute of Carcinogenesis for Cancer Research Centre in Moscow, Russia

Vladimir Janout,

Department of Preventive Medicine at Palacky University in Olomouc, Czech Republic

\footnotetext{
Corresponding Author: Sara Karami, MPH, 6120 Executive Blvd, Rockville, MD 20852, Telephone: (703) 862-2486, Fax: (301) 496-9146, karamis@mail.nih.gov.

There Are No Financial Disclosures for Any of the Above Authors
} 
Helena Kollarova,

Department of Preventive Medicine at Palacky University in Olomouc, Czech Republic

Vladimir Bencko,

Institute of Hygiene and Epidemiology at Charles University in Prague, Czech Republic

Ivana Holcatova,

Institute of Hygiene and Epidemiology at Charles University in Prague, Czech Republic

\section{Marie Navritalova, \\ Department of Cancer Epidemiology and Genetics at the MasarykMemorial Cancer Institute in Brno, Czech Republic}

Neonila Szeszenia-Dabrowska,

Department of Epidemiology at the Institute of Occupational Medicine in Lodz, Poland

Dana Mates, and

Institue of Public Health in Bucharest, Romania

Jan P. Gromiec

Nofer Institute of Occupational Medicine at the Department of Chemical Hazards in Lodz, Poland

\section{Abstract}

Background-Recent findings indicate that vitamin D obtained from ultraviolet (UV) exposure may reduce the risk of a number of different cancers. Vitamin D is metabolized to its active form within the kidney, the major organ for vitamin D metabolism and activity. Since both the incidence of renal cell cancer and prevalence of vitamin D deficiency have increased over the past few decades, this study sought to explore whether occupational UV exposure was associated with renal cell carcinoma (RCC) risk.

Methods-A hospital-based case-control study of 1,097 RCC cases and 1,476 controls was conducted in four Central and Eastern European countries. Demographic and occupational information was collected to examine the association between occupational UV exposure and RCC risk.

Results-A significant (24\%-38\%) reduction in RCC risk was observed with increasing occupational UV exposure among male participants. No association between UV exposure and RCC risk was observed among female participants. When analyses were stratified by latitude as another estimate of sunlight intensity, a stronger (71\%-73\%) reduction in RCC risk was observed between UV exposure and cancer risk among males residing at the highest latitudes.

Conclusion-The results of this study suggest that among males there is an inverse association between occupational UV exposure and renal cancer risk. Replication studies are warranted to confirm these results.

\section{Keywords}

RCC; renal cell carcinoma; renal cancer; kidney cancer; vitamin D; UV; UVB; sunlight; occupational exposure; occupational sunlight exposure

\section{Introduction}

The incidence of renal cell carcinoma (RCC), the main form of kidney cancer, has increased both in the United States (U.S.) and globally over the past two decades. ${ }^{1,2}$ Reasons for this increase remain speculative; however, reduced vitamin $\mathrm{D}$ could be a contributing factor. Recent large scale epidemiological studies, many of them ecological, have shown linear 
inverse associations between solar ultraviolet (UV) B exposure and incidence and/or mortality rates for breast, colorectal, ovarian, and prostate cancer and Hodgkin and nonHodgkin lymphoma (NHL). ${ }^{3,4}$ Anti-carcinogenic properties of vitamin D include inhibition of clonal tumor cell proliferation, induction of immune cell differentiation and apoptosis, and decreased angiogenesis. ${ }^{5,} 6$ Yet, throughout the U.S. and Europe, widespread vitamin D deficiency/insufficiency continue to be a problem for all age groups because of inadequate vitamin $\mathrm{D}$ intake and decreased sun exposure, possibly due to a sedentary lifestyle. ${ }^{4}$

Vitamin D is a fat-soluble vitamin found in food and synthesized in the skin upon exposure to solar UVB rays. ${ }^{7}$ Fortified foods, such as milk, butter, and cereal are common sources of vitamin D in the U.S. and Canada. However, in most countries, very few foods naturally contain significant amounts of vitamin $\mathrm{D}$. The majority of individuals obtain most of their vitamin D though sunlight exposure. ${ }^{7}$ In general, UVB exposure accounts for approximately $90 \%$ of 1,25-dihydroxyvitamin $\mathrm{D}\left(1,25(\mathrm{OH})_{2} \mathrm{D}_{3}\right)$ levels, the biologically active form of vitamin D. ${ }^{4,8}$ After vitamin $\mathrm{D}$ is produced in the skin or consumed in food, the vitamin is hydroxylated in the liver and subsequently in the kidney to form $1,25(\mathrm{OH})_{2} \mathrm{D}_{3} \cdot{ }^{7-11}$

Although both the incidence of renal cell cancer and prevalence of vitamin D deficiency have increased over the past two decades, the relationship between UVB exposure and kidney cancer has not been explored. $1,9,10$ Given the widespread public health interest in vitamin $\mathrm{D}$ and its potential cancer prevention promise, it is important to investigate the relationship between vitamin $\mathrm{D}$ and cancer risk, particularly renal cell carcinoma (RCC) since the kidney is the major organ for vitamin D metabolism and activity, and calcium homeostasis. ${ }^{9-11}$ In the present study, we investigated the association between estimated exposure to occupational sunlight and RCC risk in one of the largest, multi-centered renal case-control studies conducted to date in Central and Eastern Europe, an area with one of the highest rates of RCC in the world ${ }^{2}$ and an area were fortification of foods with vitamin D is not a common practice.

\section{Material and Methods}

\section{Study Population}

From 1999 through 2003, a hospital-based case-control study of RCC was conducted in seven centers in four countries of Central and Eastern Europe (Moscow, Russia; Bucharest, Romania; Lodz, Poland; and Prague, Olomouc, Ceske-Budejovice, and Brno, Czech Republic). Cases, aged 20-88 years, included patients with newly diagnosed histologically confirmed RCC (IDC-O-2 codes C64) who had lived in the study areas for at least one year and were interviewed within three months of diagnosis. RCC tumors were histologically confirmed at the National Cancer Institute (NCI) by a world expert in renal tumor pathology. Frequency-matched to cases on age (+/- 3 years), sex, and place of residence, controls were selected from patients admitted to participating hospitals for diagnoses unrelated to smoking or urological disorders (diseases of the kidneys or urinary tract) with the exception of benign prostatic hyperplasia. No single disease made up more than $20 \%$ of the control group. Some controls were also recruited in parallel for studies of lung and head and neck cancers. ${ }^{12}, 13$ All participants were of Caucasian descent. The final number of participants for analysis in this study included 1,097 renal cancer cases and 1,476 controls. The response rates across study centers for study participation ranged from $90.0 \%$ to $98.6 \%$ for cases and from $90.3 \%$ to $96.1 \%$ for controls. All subjects provided written informed consent. This study was approved by the institutional review boards of all participating centers. 


\section{Occupational Exposure Assessment}

Interviewers were trained in each center to perform face-to-face interviews of cases and controls during hospitalization using standard questionnaires. The questionnaire covered basic demographic characteristics, family history of cancer, history of tobacco consumption, and dietary habits. Lifetime occupational information for jobs of $\geq 12$ months duration was also ascertained through the use of a general occupational questionnaire. Data collected for each job included title, detailed tasks, and type of employer, as well as year of beginning and ending employment.

Job titles were coded by local industrial hygienists or occupational health experts, blinded to case-control status, according to the International Standard Classification of Occupation 1968 version (ISCO-68). ${ }^{14,}{ }^{15}$ Industries were similarly coded according to the Statistical Classification of Economic Activities of the European Community, 1999 version (NACE-99). ${ }^{15,} 16$ Both the ISCO and NACE coding systems are regularly utilized in occupational epidemiological studies conducted throughout Europe for occupation and industry classifications. ${ }^{17}$

Based on the ISCO coded job and NACE coded industry titles, a job exposure matrix (JEM) was created to categorize frequency, duration, confidence, and intensity of occupational sunlight exposure for each study participant. Frequency of exposure was estimated by the percentage of time in an 8 hour day during which exposure was possible: $<30 \%$ ( 0 to $<2.4$ hours), $30-69 \%$ ( 2.4 to 5.5 hours), and $\geq 70 \%$ ( $>5.5$ hours or more) of the time. To compute sunlight exposure across jobs that had different frequencies of exposure, frequency weights of $(0.15,0.50$, and 0.85$)$ were assigned to the three categories, corresponding to the midpoint of the range of each category. Level of confidence of occupational sunlight exposure for each job was coded in three categories as "possible" $(<40 \%)$, "probable" (40-90\%), or "certain" (>90\%), based on the likely tasks and location (indoor, outdoor) of the job, representing the degree of confidence in our assignment for frequency of exposure. Intensity of exposure was coded as "high" for participants suspected to be exposed to strong ultraviolet light reflected from the sea and for agricultural workers and outdoor occupations in a rural setting or "low" for participants suspected to be exposed to weak ultraviolet light (all other jobs). Intensity of sunlight exposure was assumed to be twice that for jobs rated "high" (two units) compared to jobs rated "low" (one unit). 18, 19

To assure high quality exposure assessment, the assignments of probability, frequency, and intensity of occupational sunlight exposure categories were reassessed by two industrial hygiene experts (MD, PS) at the NCI for each job held by each participant. Inter-rater agreement for the probability, frequency, and intensity of occupational sunlight exposure was calculated using Cohen's kappa statistic. ${ }^{20}$

\section{Statistical Analysis}

Several measures of occupational sunlight exposure were assessed:

- cumulative exposure (low exposure-unit-years) across all jobs, calculated as duration (years) * frequency midpoint * intensity of exposure (units) for each job and summed over jobs;

- frequency-adjusted duration of exposure in years across all jobs, calculated as duration (years) * frequency midpoint for each job and summed over jobs;

- frequency-adjusted duration of exposure in years among participants who held only low intensity jobs, (excluding participants who held only high intensity or both high and low intensity jobs); and 
- frequency-adjusted duration of exposure in years among participants who held any high intensity jobs (excluding participants who held only low intensity jobs).

Given the substantial variability observed for each of the estimate measures of occupational UV exposure by gender, no overlap in exposure-response categories could be assessed. Therefore, categorical exposure metrics were used to evaluate exposure-response relationships with occupational exposure based on tertiles of exposure levels among all controls, all male controls, and all female controls. However due to small numbers, frequency-adjusted duration of exposure among participants who held any high intensity jobs was evaluated dichotomously by comparing the first two (lowest) tertiles of duration to the third (highest) tertile. Unfortunately, due to small numbers, analysis for frequencyadjusted duration of exposure for subjects with only high intensity jobs (excluding participants who held only low intensity or both high and low intensity jobs) were not possible.

Subgroup analyses were examined by sex, median age, body mass index (BMI), family history of cancers, hypertensive status, smoking status, and restricted to jobs assigned a high confidence (probable or certain) in the occupational sunlight exposure assessment. Since the exposure metrics for nearly all ( $>98 \%$ ) occupations were assigned a high confidence, analyses are presented only for this high confidence subset. Subgroup analyses were also performed by latitude/study center (Russia $\left(55.8^{\circ} \mathrm{N}, 37.6^{\circ} \mathrm{E}\right)$, Poland $\left(51.6^{\circ} \mathrm{N}, 19.5^{\circ} \mathrm{E}\right)$, Czech Republic $\left(49.2^{\circ}-50.1^{\circ} \mathrm{N}, 14.4^{\circ}-17.3^{\circ} \mathrm{E}\right)$, and Romania $\left(44.4^{\circ} \mathrm{N}, 26.1^{\circ} \mathrm{E}\right)$ ) as another estimate of sunlight intensity. Exploratory analyses of associations between UV exposure and other relevant variables, BMI, self-reported hypertension, education, and smoking status, were also evaluated for potential confounding.

Odds ratios (OR) and $95 \%$ confidence intervals $(95 \% \mathrm{CI})$ were calculated to estimate RCC risk and association with occupational sunlight exposure, using unconditional logistic regression models adjusting for sex, age, center, smoking status (never, ever), BMI, and selfreported hypertensive status (no, yes). Logistic regression was performed and tests for linear trends using continuous variables were calculated to estimate RCC risk by occupational exposure. Interactions were tested comparing regression models with and without interaction terms using a likelihood ratio test (LRT). All analyses were conducted in STATA 9.0 unless otherwise specified (STATA Corporation, College Station, TX).

\section{Results}

Inter-rater agreement for assessment of intensity of occupational sunlight exposure, using Cohen's kappa statistic, was very good (kappa $=0.82)$. The agreement for frequency of occupational sunlight exposure was good (kappa $=0.73$ ). Confidence in the exposure assessment, coded in three categories as "possible," "probable," or "certain" had a fair interrater agreement $(\mathrm{kappa}=0.48)$; however, when probability of exposure was assessed as low $(<40 \%)$ versus high $(\geq 40 \%)$, inter-rater agreement was better (kappa $=0.65)$.

A description of study participants and known RCC risk factors is provided in Table 1. Cases and controls were comparable in age and education level, but cases were more likely to be female and were more likely to have an excess BMI $\left(>30 \mathrm{~kg} / \mathrm{m}^{2}\right)$ and hypertension. The association with smoking was not observed after adjustment for age, BMI, hypertension, study center, and sex. ${ }^{21}$

No significant ( $\mathrm{p}$-trend $\geq 0.05$ ) patterns in association for occupational sunlight exposure were seen for all participants. Significant linear inverse associations with RCC were, however, observed among males occupationally exposed to sunlight (Table 2). Decreased RCC risk and significant exposure-response relationships were observed among male 
participants in the highest tertile for cumulative exposure $(\mathrm{OR}=0.76 ; 95 \% \mathrm{CI}=0.58-1.00$; $\mathrm{p}$ trend $=0.05)$, frequency-adjusted duration of exposure $(\mathrm{OR}=0.76 ; 95 \% \mathrm{CI}=0.58-0.99$; $\mathrm{p}-$ trend=0.04), and frequency-adjusted duration of exposure among subjects who only held low intensity jobs $(\mathrm{OR}=0.62 ; 95 \% \mathrm{CI}=0.45-0.85 ; \mathrm{p}$-trend=0.003). A non-significant decrease in RCC risk was observed among male participants who held any high intensity jobs (p-trend $=0.60$ ). No associations were observed between RCC risk and occupational sunlight exposure for female subjects.

As another estimate of sunlight intensity, analyses were stratified by latitude/study center. No statistically significant trends were observed when the data were stratified by latitude/ study center, except among males residing in Russia. Although an inverse association between RCC risk and occupational UV exposure was observed among male participants at other study centers (data not shown), only Russian males showed a significantly strong and linear inverse association with increasing occupationally UV exposure and RCC risk (Table 3). Specifically, significant exposure-response relationships and interactions were seen for cumulative exposure ( $\mathrm{p}$-trend $<0.001 ; \mathrm{p}$-interaction $=0.001$ ), frequency-adjusted duration of exposure (p-trend $<0.001$; $\mathrm{p}$-interaction= 0.001 ), and duration of exposure among subjects who only held low intensity jobs (p-trend $<0.001$; p-interaction $=0.0001$ ). These associations were more pronounced than seen for all males combined. No association was observed for any high intensity jobs and RCC risk. No association was observed among female participants.

\section{Discussion}

To our knowledge, this is the first case-control study to show evidence of an inverse association between occupational UV exposure and RCC risk. The inverse association between occupational UV exposure and RCC risk was observed only among males. Reduced RCC risk among males was significantly and linearly observed for increasing cumulative exposure, frequency-adjusted duration of exposure, and frequency-adjusted duration of exposure for only low intensity jobs. No association between occupational UV exposure and RCC risk was observed among female participants; hypertension, smoking, and excess BMI also showed no association between UV exposure and RCC risk among females. When analyses were stratified by latitude of study center as another estimate of UV intensity, a stronger inverse association between UV exposure and RCC risk was observed among males from Moscow, the study center expected to have the lowest intensity UV exposure compared to the other centers located at lower latitudes.

The results of our study are supported by other epidemiological kidney cancer studies. In general, ecological studies investigating the association between cancer risk and sun exposure have reported an inverse relationship between kidney cancer incidence and mortality risk and UVB exposure. ${ }^{22-26}$ Recently, Mohr and colleagues examined the association between UVB exposure and renal cancer risk in 175 countries using latitude and solar UVB irradiance. ${ }^{26}$ The highest renal cancer incidence rates were found in countries situated at the highest latitudes (p-value $<0.01$ in both men and women). ${ }^{26}$ Furthermore, an occupational cohort study exploring the relationship between UV exposure and cancer risk among Swedish male construction workers found that participants exposed to the highest level of occupational sunlight had reduced kidney cancer risk ( $\mathrm{OR}=0.7 ; 95 \% \mathrm{CI}=0.4-1.00)$.

${ }^{27}$ Similar inverse associations have been reported between occupational sunlight exposure and other cancers, such as diffuse large $\mathrm{B}$-cell lymphoma $(\mathrm{OR}=0.72 ; 95 \% \mathrm{CI}=0.54-0.97)$, follicular lymphoma (p-trend $=0.04)$, and rectal cancer $(\mathrm{OR}=0.62 ; 95 \% \mathrm{CI}=0.42-0.93) .{ }^{28}$, 29 
One explanation for the inverse association between UV and cancer is the hypothesis that sunlight exposure increases production of vitamin D in the skin that travels through the blood to other sites in the body. This is particularly important with regards to kidney cancer, because the kidney is the major organ responsible for vitamin D metabolism and activity, and calcium homeostasis. ${ }^{9-11}$ Vitamin D and its analogues are thought to suppress tumor activity by inhibiting clonal tumor cell proliferation and the G1 cell cycle arrest phase, inducing immune cell differentiation and apoptosis, and decreasing angiogenesis. ${ }^{5,}, 6,30,31$

In this study, we observed significant inverse trends only among males with low intensity occupations and not among males with high intensity UV exposure. This finding was corroborated when we stratified our analysis by study center/latitude as an estimate of UV intensity. Significant trends were observed among Russian males expected to have the lowest intensity UV exposure. The lack of an apparent effect among subjects with high UV intensity exposure can be explained considering that high intensity UV exposure, which usually results at lower latitudes, can result in sufficient amounts of vitamin D in a short amount of time, whereas longer exposure time is necessary if UV intensity is lower. After saturation, equilibrium is reached and furthermore, UV exposure no longer results in the formation of additional vitamin $\mathrm{D}$ and actually starts to degrade the vitamin through photodegradation. ${ }^{32}$ For example, most healthy Caucasians can generate sufficient amounts of vitamin D with as little as 10 to 20 minutes of high intensity sun exposure to unprotected skin. ${ }^{28}$ One possibility is that, in lower latitudes with higher intensity sunlight exposures, there may not be sufficient contrast in vitamin levels across groups with varying frequency of exposures because most people are already exposed beyond this level of saturation through everyday, non-occupational activities. For the most part, individuals acquire increasing UV radiation doses with decreasing latitude. ${ }^{17}$ Around the world at mid-latitude $\left(30^{\circ}-50^{\circ} \mathrm{N}\right)$ the increase in erythemally effective UV radiation is between $3 \%$ and $3.6 \%$ for every degree of latitude towards the equator. ${ }^{17}$ Therefore individuals regardless of their occupations will obtain greater UVB exposure if they live or work at lower latitudes. In other words, occupational sunlight exposure may be relatively more important in higher latitudes with lower intensity UVB because residents there are less likely to reach the threshold of adequate sunlight exposure outside of work. ${ }^{32}$ Alternatively, the lack of association among high intensity exposure occupations, (such as farmers and gardeners) may be influenced by other carcinogenic co-exposures in this group. For instance, we have already reported increased kidney cancer risk among pesticide-exposed individuals that was highest among GSTM1/GST1 active subjects in this study. ${ }^{33}$

The association between occupational UV exposure and RCC risk was observed among males and not females in this study. Gender differences related to UV sensitivity and cancer risk have been demonstrated in other studies but it is not known whether they are real, due to due to hormonal differences between sexes, or caused by differential- misclassification due to behavior differences. ${ }^{34,35}$ Males and females may differ biologically in their response to UV exposure. Laboratory studies suggest that there are gender related hormonal differences that may play a role in responses to acute UVB exposure as well as UV-induced tumor development. ${ }^{36,37}$ In our study, no gender differences were observed between UV exposure and tumor histological subtype or grade (data not shown). However, several studies have observed gender differences related to UV exposure and cancer risk; though results have been inconsistent. A recent European case-control study that found an inverse association between UV radiation and lymphoma risk and a significant interaction between sex and skin sensitivity (tendency to sunburn) was observed with NHL risk. Specifically, female participants experienced higher risks of NHL with increasing skin sensitivity (p-trend $<0.001$; p-interaction $=0.02$ ) compared with male participants. ${ }^{28}$ Similarly, elevated NHL risk was reported among females but not among males with outdoor occupations in a large English cohort study that included 401 NHL male and 27 NHL female cases. ${ }^{38}$ Gender 
differences were also observed in an Australian occupational case-control study that found an inverse association between occupational UV exposure and risk of glioma in females $(\mathrm{OR}=0.54 ; 95 \% \mathrm{CI}=0.27-1.07)$ while there was a positive association among males $(\mathrm{OR}=$ $1.60 ; 95 \% \mathrm{CI}=0.95-2.69) .{ }^{39}$ Alternatively, gender differences may also be due to misclassification of occupational exposure due to behavioral differences, such as a higher tendency for females to use sunscreen on a regular basis and the ability of males to work outdoors while shirtless. ${ }^{40}$ Around the world (with a few exceptions) males generally tend to go outside more frequently, spend more time outdoors and therefore acquire higher UV doses than females. ${ }^{17}$ Occupational exposures may be more precise for males than for females. Historically, males have had occupations that require them to spend more time outdoors in the sun compared with females. ${ }^{36,41,42}$ It is noteworthy that in this study, occupational exposure levels estimated among females were considerably less than among males and we did not ask questions specifically related to recreational exposures among either sex. Lastly, we cannot completely rule out the possibility that these differences are due to chance.

Strengths of our study include a large sample size, a high participation rate, inclusion of only histologically confirmed cancers, and the use of both the ISCO and NACE coding systems to assign individual-specific exposure information. While confirmation with actual measurements of sunlight exposure would have been ideal, inter-rater agreement scores confirmed consistent exposure assessment for frequency, intensity, and confidence (based on two categories) of occupational sunlight exposure. However, the reliability of our assessment for confidence of occupational sunlight exposure, based on three categories, was not as precise. Several other limitations that are inherent to our study include inability to obtain data regarding recreational exposure to UV light, history of sunburns, use of tanning beds, and sunbathing activities, which have been associated with other cancers. Data regarding temporal changes in sunlight exposure was also not available. Failure to measure all sources of sunlight exposure or take into account seasonality or exact location/latitude of occupations, which would have allowed for a more accurate intensity variable, may have led to misclassification of sunlight exposure and/or biased our results towards the null. Secondly, while participants were primarily of Central European descent, information on hair color, eye color, tanning ability, use of sunscreen or personal protective equipment, such as hats, gloves, long pants, etc. were not ascertained. While we were able to control for known RCC risk factors, such as hypertension, smoking, and BMI, other potential risk modifiers (i.e. genetics, other exposures) were not considered. Although dietary intake of foods that naturally contain significant amounts of vitamin D account for less than $10 \%$ of vitamin D levels, ${ }^{43}$ dietary intake frequency of foods naturally rich in vitamin D (liver, and freshwater, saltwater, and total fish consumption) were considered in this study and no association was observed overall or by gender (data not shown) ${ }^{44,45}$; unfortunately, no information was available on dietary supplement use which may have confounding results due to healthy dietary or lifestyle patterns. Additional limitations of our study include nondifferential inaccurate or incomplete recall of all occupational histories, non-differential exposure misclassification, and the use of hospital-based controls, which may not represent the general non-diseased reference population. While this study had sufficient statistical power to detect relatively small associations, only a small subset of cases and controls had only high intensity jobs or were residences of particular study centers, thus, limiting the precision of our associations within these subgroups and increasing the potential of observing results due to chance.

To our knowledge, this is the first and largest occupational case-control study to investigate the association between UV exposure and RCC risk. Our findings, supported by most UV/ cancer studies, demonstrated that occupational UV exposure is associated with reduced renal cancer risk among males. Additional studies that consider recreational UV exposure and 
behavioral differences, in an effort to reduce exposure misclassification, are warranted, particularly among females.

\section{References}

1. Kimball S, Fuleihan Gel-H, Vieth R. Vitamin D: a growing perspective. Crit Rev Clin Lab Sci. 2008; 45(4):339-414. [PubMed: 18568854]

2. International Agency for Research on Cancer. GLOBACAN. [June 12, 2008]. (http://www-dep.iarc.fr/)

3. Grant WB, Garland CF, Gorham ED. An estimate of cancer mortality rate reductions in Europe and the US with 1,000 IU of oral vitamin D per day. Recent Results Cancer Res. 2007; 174:225-234. [PubMed: 17302200]

4. Calvo MS, Whiting SJ, Barton CN. Vitamin D intake: a global perspective of current status. J Nutr. 2005; 135(2):310-316. [PubMed: 15671233]

5. Ordonez-Moran P, Larriba MJ, Pendas-Franco N, Aguilera O, Gonzalez-Sancho JM, Munoz A. Vitamin D and cancer: an update of in vitro and in vivo data. Front Biosci. 2005; 10:2723-2749. [PubMed: 15970529]

6. Trump DL, Hershberger PA, Bernardi RJ, Ahmed S, Muindi J, Fakih M, Yu WD, Johnson CS. Antitumor activity of calcitriol: pre-clinical and clinical studies. J Steroid Biochem Mol Biol. 2004; 89-90(1-5):519-526. [PubMed: 15225831]

7. National Institutes of Health; [December 25, 2008]. Dietary Supplement Fact Sheet: Vitamin D. Archived from the original on 2007-09-10. (http://www.webcitation.org/5R15u0LB5)

8. John EM, Schwartz GG, Koo J, Wang W, Ingles SA. Sun exposure, vitamin D receptor gene polymorphisms, and breast cancer risk in a multiethnic population. Am J Epidemiol. 2007; 166(12): 1409-1419. [PubMed: 17934201]

9. Norman AW. Sunlight, season, skin pigmentation, vitamin D, and 25-hydroxyvitamin D: integral components of the vitamin D endocrine system. Am J Clin Nutr. 1998; 67(6):1108-1110. [PubMed: 9625080]

10. Matsuoka LY, Wortsman J, Haddad JG, Kolm P, Hollis BW. Racial pigmentation and the cutaneous synthesis of vitamin D. Arch Dermatol. 1991; 127(10):536-538. [PubMed: 1848745]

11. Deeb KK, Trump DL, Johnson CS. Vitamin D signaling pathways in cancer: potential for anticancer therapeutics. Nat Rev Cancer. 2007; 7(9):684-700. [PubMed: 17721433]

12. Scélo G, Constantinescu V, Csiki I, et al. Occupational exposure to vinyl chloride, acrylonitrile and styrene and lung cancer risk. Cancer Causes Control. 2004; 15(5):445-452. [PubMed: 15286464]

13. Hashibe M, Boffetta P, Zaridze D, et al. Contribution of tobacco and alcohol to the high rates of squamous cell carcinoma of the supraglottis and glottis in Central Europe. Am J Epidemiol. 2007; 165(7):814-820. [PubMed: 17244634]

14. ILO. International standard classification of occupations (ISCO). Rev. Ed.. Geneva: 1968.

15. Durusoy R, Boffetta P, Mannetje A, et al. Lung cancer risk and occupational exposure to meat and live animals. Int J Cancer. 2006; 118(10):2543-2547. [PubMed: 16353144]

16. Eurostat. NACE Rev, 1: statistical classification of economic activities in the European community. Luxembourg: 1996.

17. Mannetje A, Kromhout $\mathrm{H}$. The use of occupation and industry classifications in general population studies. Int J Epidemiol. 2003; 32(3):419-428. [PubMed: 12777430]

18. Godar DE. UV doses worldwide. Photochem Photobiol. 2005; 81(4):736-749. [PubMed: 15819599]

19. Mech M, Koepke P. Model for UV irradiance on arbitrarily oriented surfaces. Theor Appl Climatol. 2004; 77:151-158.

20. Carpenter CR. Kappa statistic. CMAJ. 2005; 173(1):15-16. [PubMed: 15997024]

21. Hunt JD, van der Hel OL, McMillan GP, Boffetta P, Brennan P. Renal cell carcinoma in relation to cigarette smoking: meta-analysis of 24 studies. Int J Cancer. 2005; 114(1):101-108. [PubMed: 15523697] 
22. Boscoe FP, Schymura MJ. Solar ultraviolet-B exposure and cancer incidence and mortality in the United States, 1993-2002. BMC Cancer. 2006; 6:264. [PubMed: 17096841]

23. Grant WB. The effect of solar UVB doses and vitamin D production, skin cancer action spectra, and smoking in explaining links between skin cancers and solid tumours. Eur J Cancer. 2008; 44(1):12-15. [PubMed: 17967529]

24. Grant WB, Garland CF. Evidence supporting the role of vitamin D in reducing the risk of cancer. J Intern Med. 2002; 252(2):178-179. [PubMed: 12190894]

25. Grant WB. An estimate of premature cancer mortality in the U.S. due to inadequate doses of solar ultraviolet-B radiation. Cancer. 2002; 94(6):1867-1875. [PubMed: 11920550]

26. Mohr SB, Gorham ED, Garland CF, Grant WB, Garland FC. Are low ultraviolet B and high animal protein intake associated with risk of renal cancer? Int J Cancer. 2006; 119(11):2705-2709. [PubMed: 16981191]

27. Håkansson N, Floderus B, Gustavsson P, Feychting M, Hallin N. Occupational sunlight exposure and cancer incidence among Swedish construction workers. Epidemiol. 2001; 12(5):552-557.

28. Boffetta P, van der Hel O, Kricker A, et al. Exposure to ultraviolet radiation and risk of malignant lymphoma and multiple myeloma- a multicentre European case-control study. Int J Epidemiol. 2008; 37(5):1080-1094. [PubMed: 18511490]

29. Slattery ML, Neuhausen SL, Hoffman M, Caan B, Curtin K, Ma KN, Samowitz W. Dietary calcium, vitamin D, VDR genotypes and colorectal cancer. Int J Cancer. 2004; 111(5):750-756. [PubMed: 15252846]

30. John EM, Schwartz GG, Koo J, Van Den Berg D, Ingles SA. Sun exposure, vitamin D receptor gene polymorphisms, and risk of advanced prostate cancer. Cancer Res. 2005; 65(12):5470-5479. [PubMed: 15958597]

31. Mullin GE, Dobs A. Vitamin d and its role in cancer and immunity: a prescription for sunlight. Nutr Clin Pract. 2007; 22(3):305-322. [PubMed: 17507731]

32. Vieth R. Vitamin D supplementation, 25-hydroxyvitamin D concentrations, and safety. Am J Clin Nutr. 1999; 69(5):842-856. [PubMed: 10232622]

33. Karami S, Boffetta P, Rothman N, et al. Renal cell carcinoma, occupational pesticide exposure and modification by glutathione S-transferase polymorphisms. Carcinogenesis. 2008; 29(8):15671571. [PubMed: 18566013]

34. Broekmans WM, Vink AA, Boelsma E, et al. Determinants of skin sensitivity to solar irradiation. Eur J Clin Nutr. 2003; 57(10):1222-1229. [PubMed: 14506481]

35. Calvo MS, Whiting SJ. Prevalence of vitamin D insufficiency in Canada and the United States: importance to health status and efficacy of current food fortification and dietary supplement use. Nutr Rev. 2003; 61(3):107-113. [PubMed: 12723644]

36. Oberyszyn TM. Non-melanoma skin cancer: importance of gender, immunosuppressive status and vitamin D. Cancer Lett. 2008; 261(10):127-136. [PubMed: 18267352]

37. Zouboulis CC, Chen WC, Thornton MJ, Qin K, Rosenfield R. Sexual hormones in human skin. Horm Metab Res. 2007; 39(2):85-95. [PubMed: 17326004]

38. Newton R, Roman E, Fear N, Carpenter L. Non-Hodgkin's lymphoma and solar ultraviolet radiation. Data are inconsistent. BMJ. 1996; 313(7052):298. [PubMed: 8704554]

39. Karipidis KK, Benke G, Sim MR, Kauppinen T, Giles G. Occupational exposure to ionizing and non-ionizing radiation and risk of glioma. Occup Med (Lond). 2007; 57(7):518-524. [PubMed: 17728306]

40. Peacey V, Steptoe A, Sanderman R, Wardle J. Ten-year changes in sun protection behaviors and beliefs of young adults in 13 European countries. Prev Med. 2006; 43(6):460-465. [PubMed: 16949656]

41. Glanz K, Buller DB, Saraiya M. Reducing ultraviolet radiation exposure among outdoor workers: state of the evidence and recommendations. Environ Health. 2007; 6:22. [PubMed: 17686155]

42. Hall HI, May DS, Lew RA, Koh HK, Nadel M. Sun protection behaviors of the U.S. white population. Prev Med. 1997; 26(4):401-407. [PubMed: 9245656]

43. Holick MF. Vitamin D: Its role in cancer prevention and treatment. Progress in Biophys Mol Biol. 2006; 92(1):49-59. 
44. Karami S, Boffetta P, Rothman N, et al. Vitamin D receptor polymorphisms and renal cancer risk in Central and Eastern Europe. J Toxicol Environ Health A. 2008; 71(6):367-372. [PubMed: 18246496]

45. Karami S, Brennan P, Navratilova M, et al. Vitamin D pathway genes, diet, and risk of renal cell carcinoma. Int J Endocrinology. 2009 In Press. 


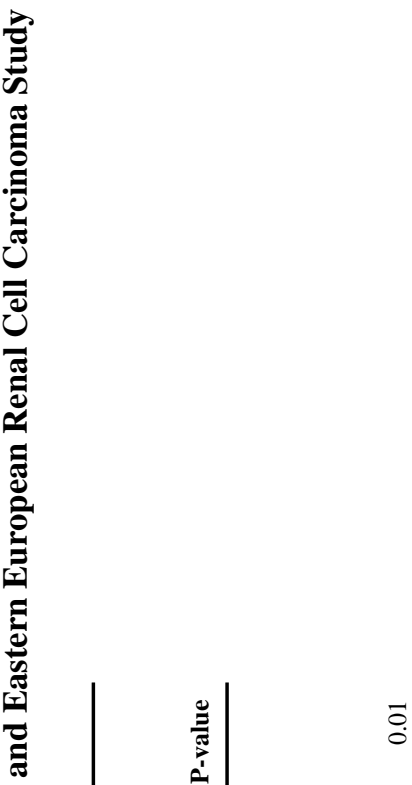

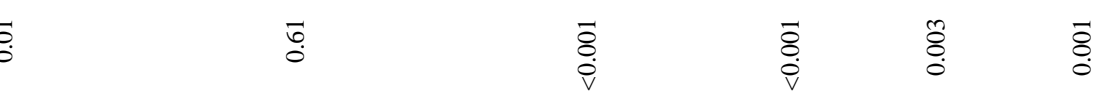

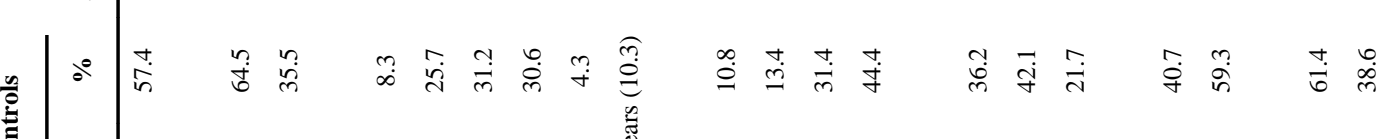

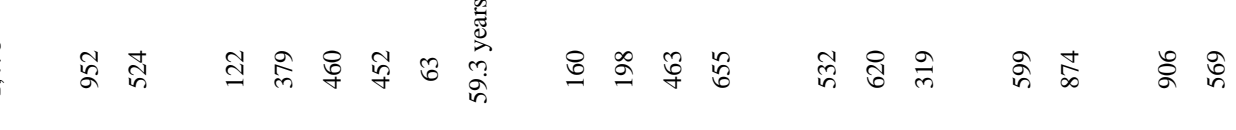

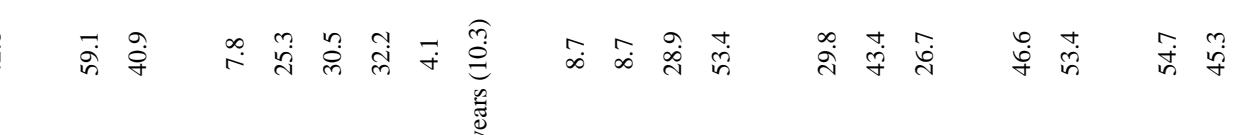

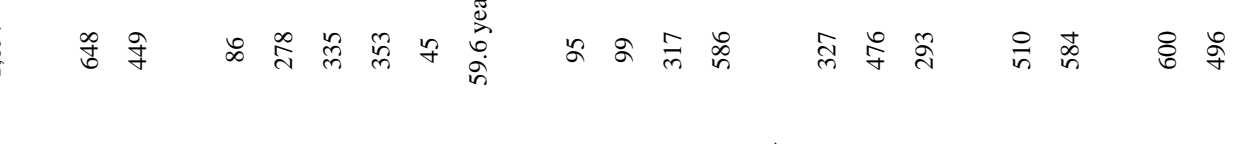

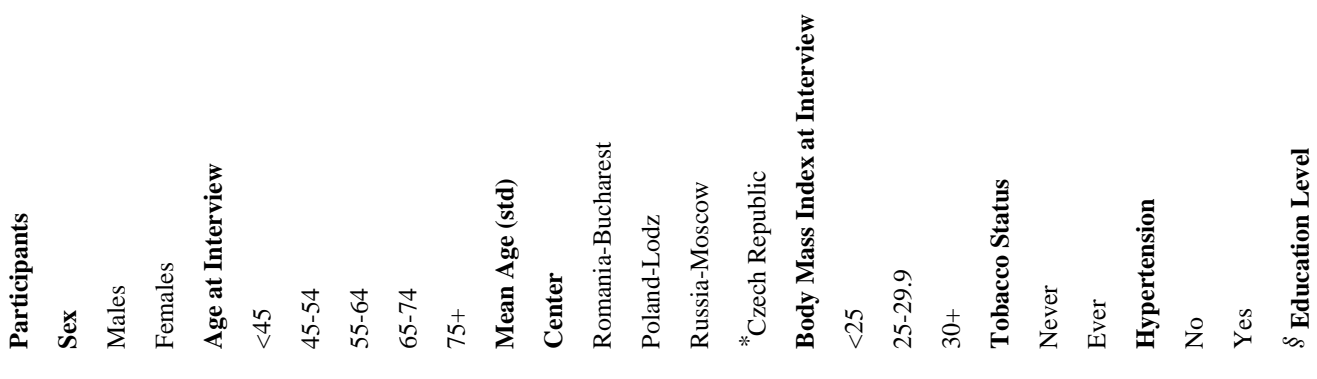




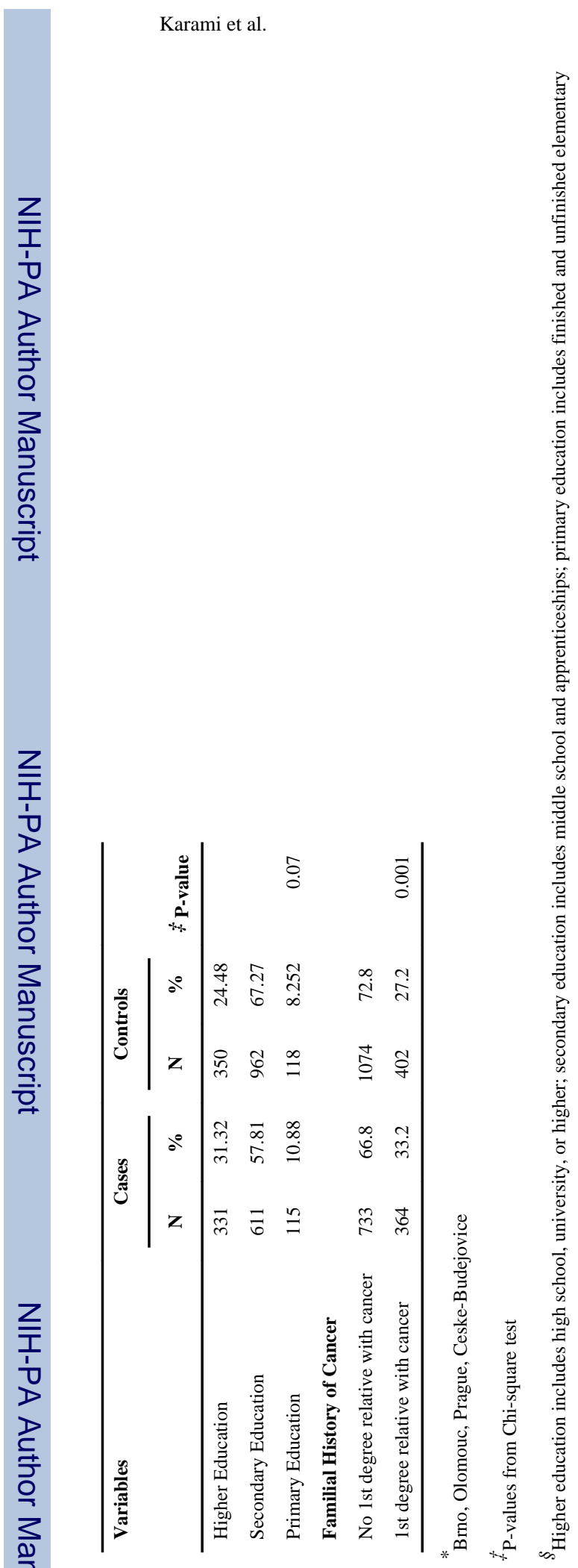

Cancer. Author manuscript; available in PMC 2011 April 15. 


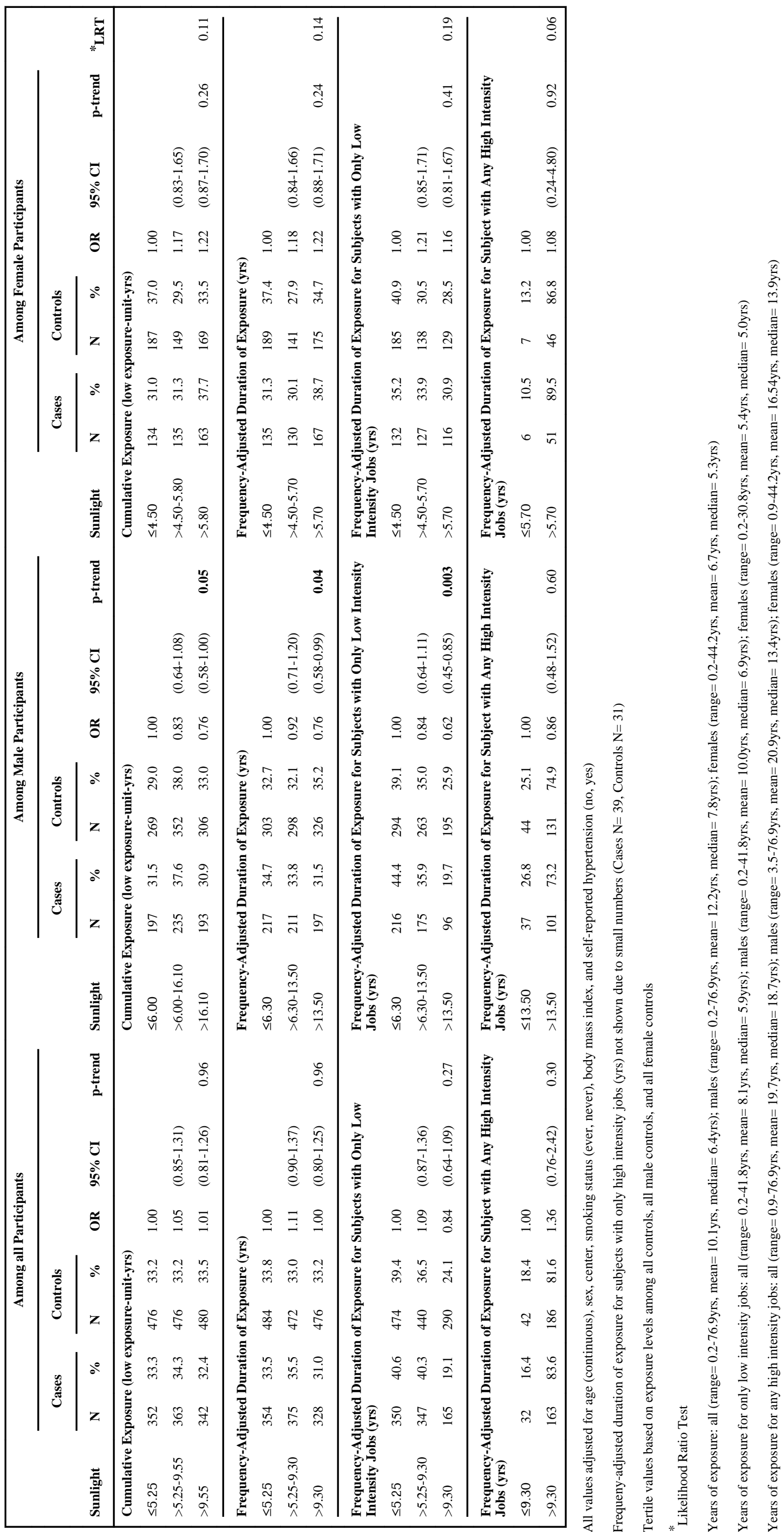




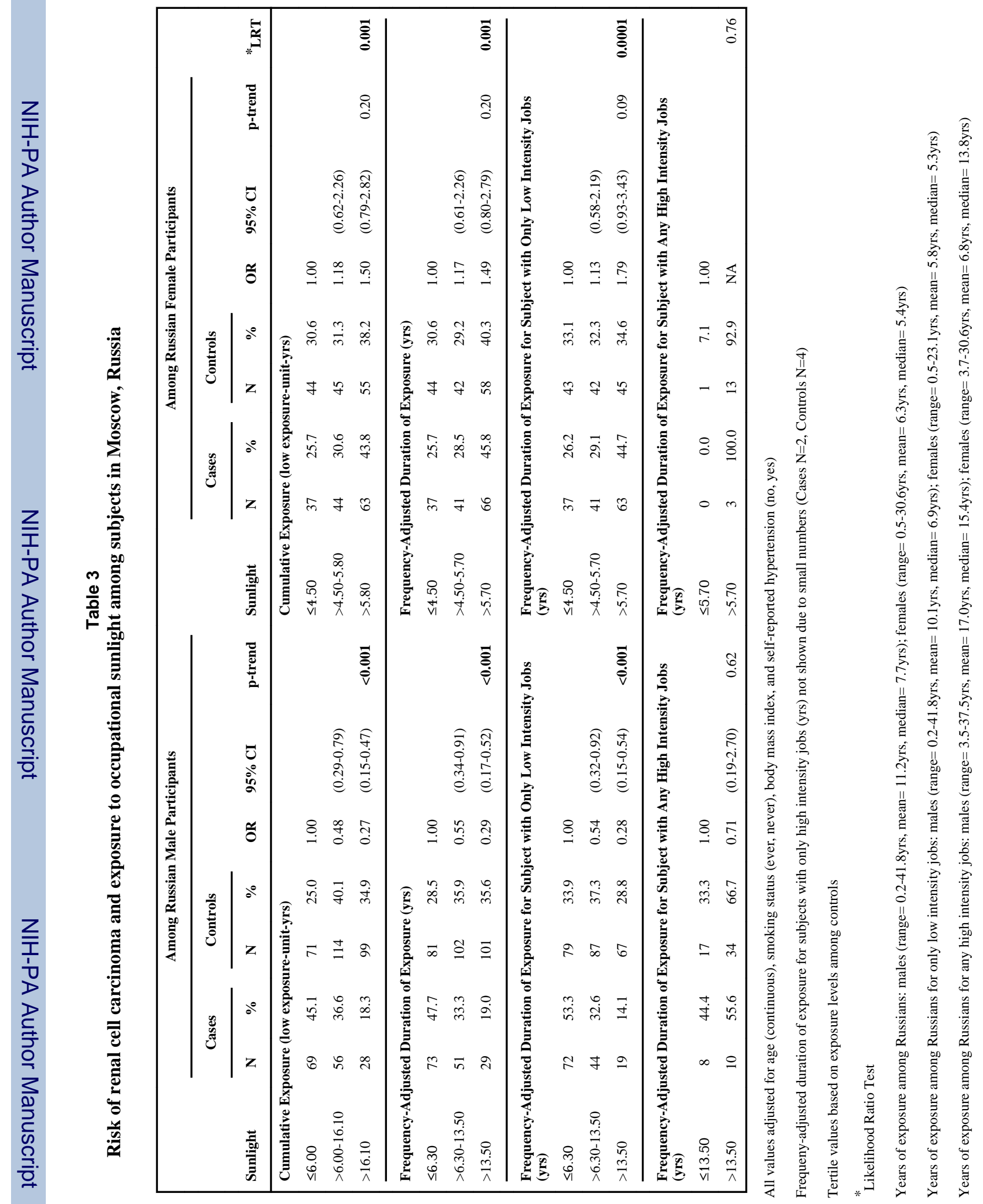

